\title{
La parola alle cose. Alcuni esempi nella poesia italiana del Novecento
}

\author{
Stefano Sasso ${ }^{1}$ \\ Published online: 2 November 2020 \\ (C) The Author(s) 2020
}

\begin{abstract}
The article discusses the presence of matter (organic as well as inorganic) in twentieth-century Italian poetry. The paper offers a diachronic survey of some exemplary authors and texts (Marinetti, Gozzano, Montale, Giudici, Caproni, and Sereni), highlighting the different utilisations of objects and things within literary texts. Objects and things define spaces and places in subjects' lives, support their identity, and document various ages and generations. The death of their owners makes things and objects lose their original purpose and find different functions with new subjects. In this context, they appear as miniature eternities always ready for a connection with new subjects.
\end{abstract}

Keywords Italian poetry $\cdot$ Eugenio Montale $\cdot$ Remo Bodei $\cdot$ Futurism $\cdot$ Train (literary motif)

\section{I-Cose e oggetti, spazio e tempo}

Si è soliti usare in modo sinonimico la parola cosa e la parola oggetto, così come inevitabilmente accadrà qualche volta anche in questo testo. Per dare però più densità concettuale a queste parole e per ampliare la percezione e il significato dei testi lirici proposti, è utile una premessa che presenti da subito le potenzialità di queste due parole e il contesto nelle quali assumono significato, cioè lo spazio e il tempo. ${ }^{1}$

L'italiano «cosa» (come in altre lingue romanze dal latino causa) si riferisce a ciò che si ritiene importante e per questa «cosa» si è pronti alla mobilitazione (da qui l'espressione «combattere per la causa» e il riferimento alla Respublica). L'equivalente concettuale di «cosa» rinvia al greco pragma, al latino res e al tedesco Sache (dal verbo «cercare», Suchen). Tali riferimenti etimologici mettono \footnotetext{
Bodei (2009) e a Pasqualotto (2018).

Stefano Sasso

stefano.sasso@romanistik.uni-halle.de

1 Martin-Luther-Universität Halle-Wittenberg, Halle, Germany
}

1 Si rinvia per l'approfondimento di questi concetti a due testi che qui si seguono per questa premessa: 
in evidenza che nella «cosa» c'è una potenzialità che riguarda la persona o una collettività. La «cosa» costituisce un nucleo di significati riconosciuti, è un contenitore di senso. In modo ancora più stringente si hanno le definizioni di «cosa stessa», come percorso obbligato del pensiero dettato dalla «cosa», che evidenzia in sé un valore di verità: ecco l'aristotelico auto to pragma e l'espressione hegeliana die Sache selbst, di cui ne è un calco, legate all'idea di vis veri, cioè che vi sia nell'uomo un istinto di verità che induce alla ricerca di significati relativi alla sostanza di qualcosa (cfr. in particolare Bodei 2009, pp. 14-15).

Il termine «oggetto», che risale alla scolastica medievale, è teoricamente vicino al greco problema, cioè un ostacolo, un impedimento che obbliga ad arrestare il cammino, così in latino il temine obicere indica un gettare contro. In sostanza l'idea di objectum (in tedesco Gegenstand, ciò che mi sta davanti) esprime una contrapposizione, «obietta» alle pretese di dominio del soggetto (Bodei 2009, pp. 19-20).

La cosa non è quindi l'oggetto, la cosa non si pone come un ostacolo che si deve affrontare e risolvere, ma rappresenta un nodo di relazioni e rinvia più alla prassi e ai rapporti umani. «Investiti di affetti, concetti e simboli che individui, società e storia vi proiettano, gli oggetti diventano cose, distinguendosi dalle merci in quanto semplici valori d'uso e di scambio o espressione di status symbol», osserva Bodei (2009, p. 22).

Il limite degli oggetti, già secondo Aristotele, è dato dallo spazio e dal luogo, che non sono quindi un semplice contenitore, ma un fattore costitutivo degli oggetti. Inoltre, «lo spazio non può esistere indipendentemente dagli oggetti nel mondo (e, dall'altra parte, nessun oggetto del mondo può esistere senza spazio)», osservando anche che «lo spazio esiste solo in rapporto a un soggetto che percepisce la realtà» (Pasqualotto 2018, pp. 161-162). Si tratta quindi di una rete di relazioni, poiché per fare un'esperienza ogni soggetto deve riferirsi allo spazio e al tempo ed è per questo motivo che per evidenziare i rapporti tra spazio, tempo e soggetto è da preferire il concetto di luogo. Il concetto di luogo definisce uno spazio determinato, ma il termine locus in latino indica anche «posto», uno spazio scelto individualmente o collettivamente, che può assorbire significati e valori. «Inoltre, più in generale, locus significa 'circostanze', il cui concetto include pertanto anche una connotazione temporale: infatti, locus ha anche il significato di 'posizione nel tempo', ossia di 'momento' e, ancor più specificatamente, di 'momento opportuno', di ciò, insomma, che i Latini chiamavano occasio, e i Greci kairòs» (Pasqualotto 2018, p. 162). Non sfugga che proprio la seconda raccolta del 1939 del premio Nobel Eugenio Montale, perno della poesia italiana del Novecento, si intitola per l'appunto Le occasioni.

Così come lo spazio non è pensabile come contenitore in cui vi sono oggetti, poiché dovrebbe essere egli stesso considerato come un oggetto indipendente dagli oggetti (mentre ne è una relazione di rapporti), così anche il tempo non è un contenitore di oggetti. «Le cose, gli eventi e noi stessi non siamo nel tempo, ma siamo tempo» (Pasqualotto 2018, p. 170). Tutto ciò condiziona ogni azione conoscitiva sia di tipo sensoriale, sia come risultato di riflessione concettuale: anche il pensare è soggetto al tempo, forma di movimento e trasformazione.

Nella prospettiva di questo scritto, le cose (e le persone a cui spesso si riferiscono) sono affidate alle parole della poesia, che (come nella pittura, nella fotografia e nel cinema) vengono trasportate in un altro spazio, che le sospende e le salva dal tempo e 
dall'oblio: le cose assumono quindi nell'uso artistico valori simbolici, «congiungono il visibile rappresentato all' invisibile assente» (Bodei 2009, p. 93). Così come appare esemplarmente in una sequenza di La dolce vita di Federico Fellini, in cui Marcello Mastroianni si trova a casa dell'intellettuale Albe Steiner, nella quale appare alla parete del suo appartamento un quadro, una Natura morta con bottiglie e bicchieri di Giorgio Morandi, e i due ne rimangono davanti parlandone: quasi una forma di mise en abyme in cui l'arte cinematografica rinvia all' arte pittorica e l'arte pittorica alle cose della vita (cfr. Sgarbi 2018, pp. 256-263). Le cose quindi sanno parlare di noi, se le lasciamo parlare nel loro proprio linguaggio, «perché su di esse ragioniamo, [...] perché-a differenza degli oggetti-non pretendiamo di servircene soltanto come strumenti o di cancellarne l'alterità e perché infine, come accade nell'arte, le sottraiamo alla loro precaria condizione [...], trasformandole in 'miniature d'eternità' che racchiudono la pienezza possibile dell'esistenza» (Bodei 2009, p. 116).

\section{II-Oggetti della modernità all'inizio del Novecento}

Il Novecento italiano della poesia si apre tra tradizione e innovazione, costituito da elementi di natura e l'innesto contrastante di elementi industriali della modernità: soprattutto treni, tramvai, automobili e biciclette. ${ }^{2}$ Gli oggetti della modernità diventano veicoli simbolici di una mutazione epocale e sono quindi soggetti ad apprezzamento o condanna. Prendiamo ad esempio il treno, presente già in testi di autori ottocenteschi, cioè in Giosuè Carducci (1835-1907) di A Satana del 1863 (in Carducci 1973), come simbolo di vittoria del progresso contro la cultura oscurantista e clericale. Il treno di Carducci diventerà però nel 1875, in un contesto più privato, di partenza e separazione da una persona cara, anche un mostro: come si può leggere in Alla stazione una mattina d'autunno in Odi barbare (1877). Sempre il treno assieme ai fili del telegrafo, anche se entrambi non nominati esplicitamente, si trovano in Giovanni Pascoli (1855-1912) nella poesia La via ferrata della raccolta Myricae (1891; in Pascoli 1981): elementi, come spesso nella sua poesia, antropomorfizzati o comunque addomesticati (i rumori del treno diventano un «femminil lamento»; i fili del telegrafo mossi dal vento si trasformano in una «immensa arpa sonora») e così assorbiti nel mondo della campagna e della tradizione. Per non dimenticare Gabriele D’Annunzio (1863-1938), si ricordi la sua esaltazione per le auto, gli aerei, nonché il possesso in privato di varie preziose automobili. ${ }^{3}$

Con la proposta del Futurismo (Fondazione e Manifesto del 1909) si è poi alla massima esaltazione dei manufatti industriali nei testi artistici, ma anche nei manifesti del movimento, come presenta lo studio dell'Accademia della Crusca che offre anche le ricorrenza dei lemmi (Accademia della Crusca 2017/18). Come si sa, in questa avanguardia tutto il repertorio degli oggetti industriali legati alla velocità, alla forza, alla dinamicità, e quindi automobili, treni, aerei diventano il nuovo

\footnotetext{
2 Per la bicicletta in testi poetici tra Otto e Novecento si segnala Matteo Pedroni (2001).

3 Si veda sul tema dei motori nella poesia internazionale l'antologia: AA.VV., Motori diVersi (AA.VV. 2001).
} 
panorama del mondo del poeta futurista. Si può qui ricordare, tra i vari testi citabili, ad esempio quello del 1908 di Filippo Tommaso Marinetti (1876-1944) dal titolo All'automobile da corsa.

Veemente dio d'una razza d'acciaio,

Automobile ebbra di spazio,

che scalpiti e fremi d'angoscia

rodendo il morso con striduli denti...

Formidabile mostro giapponese,

dagli occhi di fucina,

nutrito di fiamma

e d'olî minerali,

avido d'orizzonti e di prede siderali...

io scateno il tuo cuore che tonfa diabolicamente,

scateno i tuoi giganteschi pneumatici,

per la danza che tu sai danzare

via per le bianche strade di tutto il mondo!... [...].

(Marinetti 1908, in De Maria 1973, pp. 310-315, vv. 1-13).

Un mondo di macchine che da una parte sono vissute come delle moltiplicatrici delle qualità dinamiche dell'uomo (velocità, movimento) (cfr. Marinetti, L'Uomo moltiplicato e il Regno della Macchina (1910), in De Maria 1973, pp. 38-42) e dall'altra parte si esaltano anche in vista di un superamento dell'uomo: basti pensare anche alle proposte futuriste sulla lingua che prevedevano solo verbi all'infinito (con la sparizione quindi del soggetto) e l'eliminazione di aggettivi e avverbi (le sedi principali in cui il soggetto esprime la sua percezione del mondo) (Mengaldo 1994, pp. 206-210). Si deve inoltre aggiungere che nel Futurismo l'esaltazione di queste macchine e degli oggetti industriali è fortemente in relazione ad una prospettiva bellica, basti ricordare che nel 1915 Marinetti pubblica Guerra sola igiene del mondo (Marinetti 1915).

Negli stessi anni in cui è attivo il movimento d'avanguardia del Futurismo operano anche i poeti crepuscolari (così definiti in un articolo di Giuseppe Antonio Borgese su La Stampa del 01/09/1910), che non sono un'avanguardia organizzata in un movimento o gruppo, ma che condividono tratti tra loro comuni, pur nelle diversità dei caratteri e dello stile della loro produzione: si pensi a Sergio Corazzini, Corrado Govoni, Guido Gozzano, Aldo Palazzeschi, Marino Moretti ed altri ancora. ${ }^{4}$ In opposizione alla proposta superomistica ed estetizzante della poesia di Gabriele D'Annunzio o al mito della macchina del Futurismo di Filippo Tommaso Marinetti, i crepuscolari non affidano al poeta nessun compito, anzi negano di essere poeti nel senso tradizionale e diventano i rappresentati dell'inutilità esistenziale, dell'impotenza, della malinconia. I loro testi avranno, però, molta forza di influenzare la successiva poesia del Novecento, poiché riporteranno al centro il tema del rapporto del soggetto con la semplice realtà quotidiana fatta anche di oggetti che circondano l'esistenza dell'individuo e dove l'individuo crepuscolare, privo di

\footnotetext{
4 Per sondare il vocabolario di questi poeti e più in generale alcuni autori chiave della poesia italiana del Novecento si rinvia a Giuseppe Savoca (1995).
} 
progettualità, si sentiva già cosa tra le $\operatorname{cose}^{5}$ Tra i vari poeti crepuscolari citati, risalta la figura di Guido Gozzano per la ricchezza, la complessità e per la componente di letterarietà che caratterizza la sua poesia in forma di citazionismo di autori del passato, quasi una specie di nutrimento e di seconda natura (cfr. Bonfiglioli 1958). La poesia di Gozzano testimonia, per quanto riguarda questo studio, la funzione fondamentale degli oggetti, come in questo testo esemplare dal titolo L'amica di nonna Speranza, in La via del rifugio (1907) e poi, con qualche variante, nella raccolta più nota I colloqui (1911).

Loreto impagliato ed il busto d'Alfieri, di Napoleone

i fiori in cornice (le buone cose di pessimo gusto),

il caminetto un po' tetro, le scatole senza confetti,

i frutti di marmo protetti dalle campane di vetro,

un qualche raro balocco, gli scrigni fatti di valve,

gli oggetti col monito salve, ricordo, le noci di cocco,

Venezia ritratta a musaici, gli acquerelli un po' scialbi,

le stampe, i cofani, gli albi dipinti d'anemoni arcaici,

le tele di Massimo d'Azeglio, le miniature,

i dagherottìpi: figure sognanti in perplessità,

il gran lampadario vetusto che pende a mezzo il salone

e immilla nel quarzo le buone cose di pessimo gusto,

il cùcu dell'ore che canta, le sedie parate a damasco

chèrmisi.... rinasco, rinasco del mille ottocento cinquanta!

(L'amica di nonna Speranza, in Gozzano 1977, pp. 198-199, vv. 1-14).

Si è di fronte a una serie di oggetti elencati ed esposti ad apertura di questa poesia quasi con la funzione di immergere il lettore immediatamente nel passato, annotando però un giudizio morale che riguarda tali oggetti nell' arredo borghese dell'epoca, le buone cose di pessimo gusto, dove un lampadario moltiplica col suo quarzo all'infinito nello spazio tali oggetti. Tali oggetti inducono il poeta, e il lettore, nel passato, gli offrono un'alternativa temporale al presente, un viaggio nel tempo: rinasco del mille ottocento cinquanta!. Si presentano ad incipit di questo testo una serie di «oggetti orfani», oggetti «abbandonati dai loro precedenti proprietari, che siamo chiamati ad adottare, rifiutare o ignorare» (Bodei 2009, p. 27). Oggetti che quindi possono potenzialmente vivere più volte, congiungendo generazione a generazione, come accade con le cose di famiglia. Altro testo esemplare è La signorina Felicita ovvero la Felicità, sempre ne I colloqui (1911).

Bellezza riposata dei solai

dove il rifiuto secolare dorme!

In quella tomba, tra le vane forme

di ciò ch'è stato e non sarà più mai,

bianca bella così che sussultai,

la Dama apparve nella tela enorme:

«È quella che lasciò, per infortuni,

\footnotetext{
$\overline{5}$ Ci si permette di rinviare a Sasso 2005.
} 
la casa al nonno di mio nonno.... E noi

la confinammo nel solaio, poi

che porta pena.... L'han veduta alcuni

lasciare il quadro; in certi noviluni

s'ode il suo passo lungo i corridoi....»

Il nostro passo diffondeva l'eco

tra quei rottami del passato vano,

e la Marchesa dal profilo greco,

altocinta, l'un piede ignudo in mano,

si riposava all'ombra d'uno speco

arcade, sotto un bel cielo pagano.

Intorno a quella che rideva illusa

nel ricco peplo, e che morì di fame,

v'era una stirpe logora e confusa:

topaie, materassi, vasellame,

lucerne, ceste, mobili: ciarpame

reietto, così caro alla mia Musa!

Tra i materassi logori e le ceste

v'erano stampe di persone egregie;

incoronato delle frondi regie

v'era Torquato nei giardini d'Este.

«Avvocato, perché su quelle teste

buffe si vede un ramo di ciliegie?»

Io risi, tanto che fermammo il passo,

e ridendo pensai questo pensiero:

Oimè! La Gloria! un corridoio basso,

tre ceste, un canterano dell'Impero,

la brutta effigie incorniciata in nero

e sotto il nome di Torquato Tasso!

(La Signorina Felicita, in Gozzano 1977, pp. 184-185, vv. 133-169).

L'avvocato (altra figura ridotta attraverso la professione a puro «oggetto») e la signorina Felicita visitano il solaio della villa e lì, sepolti sotto al tetto, si trovano una massa di oggetti diversi che convivono senza più una relazione né tra loro né con il presente. Quadri che ritraggono figure femminili di famiglia, Torquato Tasso, profili greci assieme a stampe di persone «egregie» e che ora condividono lo spazio con materassi, vasellame, ceste, mobili e altri oggetti della quotidianità. Rifiuti del tempo, dice Gozzano, una soffitta che è una specie di tomba, una massa di rottami del passato dove l'oggetto prezioso ha ora lo stesso valore dell'oggetto povero, mostrando l'illusorietà dell'ordine dei valori nel tempo e delle attenzioni che a questi oggetti si è disposti a riconoscere. Sono proprio questi oggetti rifiutati dal presente, dice Gozzano, a costituire la sua Musa. L'individuo e l'oggetto posti a confronto, fino al punto di trasformare, dichiaratamente, l'individuo in oggetto, scritto in minuscolo e tutto attaccato: questa cosa vivente / detta guidogozzano (La via del rifugio, in Gozzano 1977, p. 62, vv. 35-36), un coso con due gambe / detto guidogozzano (Nemesi, in Gozzano 1977, p. 117, vv. 67-68), ne più ricordi $i$ colloqui tenuti con guidogozzano (Alle soglie III, in Gozzano 1977, p. 162, v. 34). 
Tratto caratterizzante la poesia crepuscolare di Gozzano è quindi il catalogo di oggetti tra le quali è immerso e abbandonato anche il soggetto, il quale anche nella descrizione fisica, nel colore degli occhi della donna vede l'azzurro di un piatto da cucina, un azzurro di stoviglia.

Sei quasi brutta, priva di lusinga nelle tue vesti quasi campagnole, ma la tua faccia buona e casalinga, ma i bei capelli di color di sole, attorti in minutissime trecciuole, ti fanno un tipo di beltà fiamminga....

$E$ rivedo la tua bocca vermiglia cosi larga nel ridere e nel bere, e il volto quadro, senza sopracciglia, tutto sparso d'efelidi leggiere

e gli occhi fermi, l'iridi sincere azzurre d'un azzurro di stoviglia....

(La Signorina Felicita, in Gozzano 1977, pp. 181-182, vv. 73-84).

\section{III-Eugenio Montale}

Gli studi sull'opera di Montale sono ormai enciclopedici, per cui si rinvia ad essi per gli approfondimenti, ${ }^{6}$ mentre qui si ha l'intenzione di sviluppare solamente alcuni aspetti nella prospettiva sopra delineata. Appare tipico nella poesia del più importante poeta italiano del Novecento la presenza di cose, anche di natura, che appaiono tra loro irrelate. Si veda uno dei testi più noti e forse il più antico di Montale, Meriggiare pallido e assorto del 1916, dalla sua prima raccolta Ossi di seppia (1925):

Meriggiare pallido e assorto

presso un rovente muro d'orto,

ascoltare tra i pruni e gli sterpi

schiocchi di merli, frusci di serpi.

Nelle crepe del suolo o su la veccia

spiar le file di rosse formiche

ch'ora si rompono ed ora s'intrecciano

a sommo di minuscole biche.

Osservare tra frondi il palpitare

lontano di scaglie di mare

mentre si levano tremuli scricchi

di cicale dai calvi picchi.

E andando nel sole che abbaglia

\footnotetext{
6 Si veda almeno Blasucci 2002; Contini 1974; Jacomuzzi 1978; Luperini 1992; e soprattutto: Mengaldo 1989; 1996 pp. 15-162, 357-381 (articoli da 1966 a 1973); 2000; per una più estesa bibliografia della critica e per i commenti ai testi si vedano le edizioni delle raccolte di Montale pubblicate negli Oscar Mondadori sotto la direzione di Guido Mazzoni.
} 
sentire con triste meraviglia

com'è tutta la vita e il suo travaglio

in questo seguitare una muraglia

che ha in cima cocci aguzzi di bottiglia.

(Montale 1980, p. 28).

All'elencazione degli elementi di natura, irrelati e sospesi in una percezione retta dai verbi all'infinito, in questo hortus conclusus, che è il luogo in cui si realizza la rete di relazione del soggetto, appare in chiusura un manufatto, una muraglia / che ha in cima cocci aguzzi di bottiglia. Questo materiale si impone nel testo e coagula su di sé sinteticamente il senso del testo, l'impossibilità di superare il limite imposto dalle cose, l'impossibilità di superare l'isolamento e la prigionia del soggetto che anela alla conoscenza. I testi di Montale (almeno dagli Ossi a La bufera), ricchi anch'essi di oggetti, cose, manufatti, non sono puri cataloghi come nei testi crepuscolari di Gozzano (cfr. Gozzano, dopo trent'anni (1951), in Montale 1996a, b, pp. 1270-1280), ma mostrano una potenzialità metafisica. ${ }^{7}$ Le cose si impongono al soggetto, lasciando intravedere una potenzialità di senso che però non si lascia sciogliere, non si traduce in altre parole definitive o in una glossa conclusiva, rinviando quindi il loro significato nella nominazione della cosa stessa e nell'irradiazione di sensi di cui è capace e di cui è custode. ${ }^{8}$ Così si può leggere in I limoni del 1922, sempre da Ossi di seppia:

[...] Vedi, in questi silenzi in cui le cose

s'abbandonano e sembrano vicine

a tradire il loro ultimo segreto,

talora ci si aspetta

di scoprire uno sbaglio di Natura,

il punto morto del mondo, l'anello che non tiene,

il filo da disbrogliare che finalmente ci metta

nel mezzo di una verità.

Lo sguardo fruga d'intorno,

la mente indaga accorda disunisce

nel profumo che dilaga

quando il giorno più languisce

(Montale 1980, p. 9, vv. 22-33).

Seguendo il paragrafo di apertura di questo scritto, si potrebbe dire che gli oggetti si trasformano in cose e liberano tutte le loro potenzialità di senso solo nel rapporto con il soggetto (o con il lettore), conservando nell' intimità privata di questa relazione la loro vita. Le cose con cui si convive sorreggono e inducono la nostra quotidiana percezione del mondo. Per la poesia di Montale si è fatto spesso ricorso a definizioni come «elencazione ellittica» (Jacomuzzi 1978, pp. 4-13) e «correlativo oggettivo» (usato già in un saggio di Thomas Eliot del 1919; Eliot 1921), l'idea cioè che dall'oggetto nominato deve sorgere l'emozione che si vuole suscitare nel lettore.

\footnotetext{
${ }_{7}$ Nella poesia di Montale gli oggetti acquistano «un'evidenza evocativa (e una carica gnoseologica) ignota ai crepuscolari: ma la loro collocazione culturale è appunto nel repertorio crepuscolare», Blasucci 2002, pp. 28-29.

${ }^{8}$ Secondo Bodei, le cose si mostrano con un continuo sovrappiù di significazioni, ogni cosa ha «un residuo non analizzabile, un fascio di legami insaturi e di allusioni ineffabili (non perché non si possono dire, ma perché non si finirebbe mai di dire) con ciò che ancora può essere pensato», Bodei 2009, p. 29.
} 
Seguendo le parole di Montale, l'idea diventa esplicita: «Ammesso che in arte esista una bilancia tra il di fuori e il di dentro, tra l'occasione e l'opera-oggetto bisognava esprimere l'oggetto e tacere l'occasione-spinta. Un modo nuovo, non parnassiano, di immergere il lettore in medias res, un totale assorbimento delle intenzioni nei risultati oggettivi» (Montale [1946] 1976, p. 567). Si veda, ad esempio, il senso della perdita e del disorientamento, detti perfettamente dagli oggetti nominati in $L a$ casa dei doganieri, cioè la bussola, i dadi e la banderuola, la luce della petroliera:

Tu non ricordi la casa dei doganieri

sul rialzo a strapiombo sulla scogliera:

desolata t'attende dalla sera

in cui v'entrò lo sciame dei tuoi pensieri

e vi sostò irrequieto.

Libeccio sferza da anni le vecchie mura

e il suono del tuo riso non è più lieto:

la bussola va impazzita all'avventura

e il calcolo dei dadi più non torna

Tu non ricordi; altro tempo frastorna

la tua memoria; un filo s'addipana.

Ne tengo ancora un capo; ma s'allontana

la casa e in cima al tetto la banderuola

affumicata gira senza pietà.

Ne tengo un capo; ma tu resti sola

né qui respiri nell'oscurità.

Oh l'orizzonte in fuga, dove s'accende

rara la luce della petroliera!

Il varco è qui? (Ripullula il frangente

ancora sulla balza che scoscende ...).

Tu non ricordi la casa di questa

mia sera. Ed io non so chi va e chi resta.

(In Le occasioni (1939); Montale 2011, con commento, p. 180-184).

$\mathrm{O}$ ancora, altro esempio, un altro addio, un altro treno (che si possono confrontare con la poesia sopra citata di Giosuè Carducci Alla stazione una mattina d'autunno) dove si può osservare tutta la modernità di questo testo, Addio fischi nel buio...da Le occasioni (1939), in cui a Montale basta la pura nominazione di cose per immergere il lettore nella situazione:

Addii, fischi nel buio, cenni, tosse

e sportelli abbassati. É l'ora. Forse

gli automi hanno ragione. Come appaiono

dai corridoi, murati!

-Presti anche tu alla fioca

litania del tuo rapido quest'orrida

e fedele cadenza di carioca? $\_9$

\footnotetext{
9 In Le occasioni, Montale 2011, con commento, pp. 104-106. Sempre nella stessa raccolta si segnala anche un titolo di una poesia che allude a un treno veloce: Accelerato.
} 
Altro aspetto interessante nella poesia di Montale è che gli oggetti assolvono, scaramanticamente, anche la loro funzione di porta fortuna e di amuleti, hanno cioè la funzione di sostenere la vita del soggetto nei momenti di maggiore difficoltà (cfr. Bodei 2009, pp. 28-29 e le osservazioni sugli oggetti e il linguaggio del sacro). Questo è il caso della poesia Dora Markus, ispirata a un'austriaca di origine ebrea nel tempo del nazismo e delle leggi razziali e che si trova travolta da questa tragedia (Montale 2011, pp. 63-73, con commento), in cui compare il topo bianco d'avorio, un amuleto conservato tra gli oggetti per la cura estetica della persona, la matita delle labbra, il piumino e la lima per le unghie.

[...]

Non so come stremata tu resisti

in questo lago

d'indifferenza ch'è il tuo cuore; forse

ti salva un amuleto che tu tieni

vicino alla matita delle labbra,

al piumino, alla lima: un topo bianco,

d'avorio; e così esisti!

(Montale 2011, p. 67, vv 22-28).

Un giovane poeta di quegli anni, Vittorio Sereni, in una recensione a Le occasioni, aveva perfettamente focalizzato il tratto caratteristico di queste poesie di Eugenio Montale, cogliendone l'originalità, che diventerà poi un tratto importante per la poesia italiana di altri autori più giovani, Sereni incluso. Così si può leggere nella recensione di Sereni del 1940, intitolata «In margine alle Occasioni»:

(...) la configurazione delle sue immagini, la eco delle sue parole lasciano in noi una memoria d'assoluto. Montale è il primo poeta nostro che abbia saputo rivelare, attraverso la propria intima problematicità, tutte le risorse di poesia che il nostro mondo moderno racchiude. E direi addirittura il nostro mondo fisico moderno, avvertito da altri unicamente come peso e, in quanto tale, ritenuto indegno di essere espresso; oppure ironizzato, messo davanti come l'estremo elemento di una polemica, come l'indizio di una condizione di schiavitù. Non è che tutto questo esuli completamente da Montale; perlopiù è in lui, questo nostro mondo, la base normale di ogni avvenimento poetico; spesso, un limite incantato entro cui le cose possono veramente esistere. (Sereni [1940] 1973, pp. 10-11).

Per rendere ancora più esplicita la presenza e la nominazione di oggetti e cose nella poesia di Montale, si propongono qui di seguito altri versi esemplari. Gli oggetti, in questo caso, accompagnano anche la situazione d'emergenza di salute di Drusilla Tanzi nel 1945, che diventerà la moglie di Montale nel 1963, nominata «Mosca» per gli occhiali che doveva portare per la sua forte miopia (e la presenza di occhiali è frequente nella poesia montaliana) (Savoca 1995, p. 671). In questo testo, Ballata scritta in una clinica, da La bufera (1956), la futura moglie di Montale (affetta da spondilite anchilosante) si trova sorretta fisicamente da un manichino di gesso, lì con i suoi grossi occhiali di tartaruga e sul comodino uno scaramantico bulldog di legno e una sveglia col fosforo sulle lancette, che illumina tenuemente 
la salvezza (in senso biblico) e anche il possibile trapasso dalla vita alla morte (cfr. Romolini 2012, pp. 129-139).

[...]

t'avevano chiuso di colpo

in un manichino di gesso.

Nel cavo delle tue orbite

brillavano lenti di lacrime

più spesse di questi tuoi grossi

occhiali di tartaruga

che a notte ti tolgo e avvicino

alle fiale della morfina.

[...]

Hai messo sul comodino

il bulldog di legno, la sveglia

col fosforo sulle lancette

che spande un tenue lucore

sul tuo dormiveglia,

il nulla che basta a chi vuole

forzare la porta stretta; [...].

(Ballata scritta in una clinica, in Montale 1980, pp. 209-210, vv. 13-19 e 33-39).

Piccoli oggetti della quotidianità, piccoli amuleti (il bulldog di legno, la sveglia, col fosforo sulle lancette), che sono chiamati a opporre una resistenza alla morte, a testimoniare e «a simboleggiare la dignità del continuo sforzo dell'uomo alla ricerca di un senso» (Romolini 2012, p. 139).

Fin qui, secondo l'uso nella poesia di Montale, si possono considerare gli oggetti e le cose nominate come fonti di significati, matrici che superano la loro presenza oggettiva, testimonianze di assenze, sigilli di eventi. Proprio in questa loro funzione, essi sostengono e certificano l'identità del soggetto, la sua esperienza e la sua essenza, la sua biografia. ${ }^{10} \mathrm{Nel}$ momento in cui questi oggetti o cose scompaiono, anche fisicamente, cade nell'oblio anche ciò che costituiva un supporto concreto all'identità, la testimonianza oggettiva che certificava l'identità del soggetto, il rapporto tra soggetto e oggetto si annulla. In un certo senso si capovolge la situazione sopra descritta: non si tratta più di «oggetti orfani», ma di un «soggetto orfano», che ha perso quelle cose con cui aveva istaurato un rapporto di senso. Significativa è questa poesia, L'alluvione ha sommerso il pack dei mobili (1966), presente nella quarta raccolta di Montale, Satura (1971).

L'alluvione ha sommerso il pack dei mobili,

delle carte, dei quadri che stipavano

un sotterraneo chiuso a doppio lucchetto.

Forse hanno ciecamente lottato i marocchini

Rossi, le sterminate dediche di Du Bos,

il timbro a ceralacca con la faccia di Ezra,

il Valèry di Alain, l'originale

$\overline{{ }^{10} \mathrm{Si} \text { veda per questo argomento }}$ la rivista aut aut 1985; Bodei 2002 e 2015. 
dei Canti Orfici-e poi qualche pennello

da barba, mille cianfrusaglie e tutte

le musiche di tuo fratello Silvio.

Dieci, dodici giorni sotto un'atroce morsura

di nafta e sterco. Certo hanno sofferto

tanto prima di perdere la loro identità.

Anch'io sono incrostato fino al collo se il mio

stato civile fu dubbio fin dall'inizio.

Non torba m'ha assediato, ma gli eventi

di una realtà incredibile e mai creduta.

Di fronte ad essi il mio coraggio fu il primo

dei tuoi prestiti e forse non l'hai saputo.

(L'alluvione ha sommerso il pack dei mobili, in Montale 2009, con commento, pp. 75-80).

Posta tra la prima parte e la seconda parte della raccolta, la poesia segue la parte degli Xenia, dei testi dedicati alla moglie morta ne1 1963. L'alluvione... rinvia oggettivamente all'evento dell'alluvione del 4 novembre 1966 a Firenze e quindi alla perdita definitiva di una serie di oggetti conservati in cantina. Ma la poesia allude anche allegoricamente alla crisi storica e alla crisi personale di Montale, alla fine di un mondo e delle sue basi culturali rappresentate proprio da quegli oggetti già sepolti, capaci di riapparire un'ultima volta nella memoria per poi sparire definitivamente, catturati e trasportati da un'alluvione (rappresentante metaforica della cultura di massa), come un pack che si stacca dalla banchisa polare per disperdersi (Montale 2009, p. 79). Assieme al pennello da barba, spariscono i riferimenti culturali del passato, le dediche del critico francese Charles Du Bos (1882-1939) conosciuto da Montale e rappresentante della cultura degli anni Trenta su cui Montale stesso si era formato, le lettere il timbro a ceralacca con la faccia di Ezra Pound (1885-1972) amico personale di Montale, il commento di Alain (pseudonimo di Emile-Auguste Chartier, 1868-1951) agli Charmes di Paul Valéry, il testo originale e leggendario dei Canti Orfici di Dino Campana (1885-1932), le musiche del fratello della moglie, compositore, Silvio: nemmenol' arte si salva più dalla cultura di massa, rappresentata metaforicamente dall'alluvione. Così anche il soggetto che su quegli oggetti fondava la sua formazione e la sua identità, nonostante la sofferenza e la lotta per sopravvivere, sembra travolto definitivamente da questa «alluvione». Un soggetto assediato dalle cose, come testimoniano le parole stesse di Montale in un'intervista dell' 8 aprile 1973: "Nel caso in cui l'uomo sia assediato dalle cose (è la mia attuale situazione) la voce non può dialogare che con esse, magari per tentare di esorcizzale» (Finché l'assedio dura, in Montale 1996a, b p. 1503).

Da Satura in avanti, nelle sue ultime raccolte, il vocabolario delle «cose» montaliane, diventa sempre più un «arredo» banalizzato dell'esistenza quotidiana, le bottiglie di Merlot di Hemingway, la pasta al ragù e il piatto a terra in cucina per il porcospino, le tinte del mare in un quadro che si traducono in vino, caffè e dentifricio, come da Satura in Due prose veneziane, II:

[...] l'orso Hemingway ha abboccato all'amo. 
È ancora a letto, dal pellame bucano

solo gli occhi e gli eczemi.

Due o tre bottiglie di Merlot

avanguardia del grosso che verrà.

Giù al ristorante sono tutti a tavola.

(Due prose veneziane, II, in Montale 2009, p. 268, vv: 8-12; con commento, pp. 263-269).

Sempre in Satura, in A pianterreno:

Scoprimmo che al porcospino

piaceva la pasta al ragù.

Veniva a notte alta, lasciavamo

il piatto a terra in cucina.

(A pianterreno, in Montale 2009, pp. 181-182, vv. 1-4).

E in L'arte povera da Diario del'71 e del'72:

Vino e caffè, tracce di dentifricio

se in fondo c'era un mare infiocchettabile

queste erano le tinte.

(L'arte povera, in Montale 2010, p. 16, vv. 8-10; con commento pp. 14-18).

La realtà delle cose si mostra perfettamente funzionale al senso di svuotamento simbolico che caratterizza gli anni del boom economico, con il corrispettivo consumo continuo di cibo e di oggetti sempre sostituibili con altri. Gli oggetti appaiono allora come scaricati da una loro propria valenza originale: si è di fronte quindi ad oggetti che difficilmente diventeranno cose, nel senso espresso da Remo Bodei, e quindi una materialità che ha una vita breve, oggetti che hanno nella sostituibilità e nell'essenza del consumismo il loro unico destino, facendosi specchio del destino del soggetto. Tutto si mischia, come in questo prossimo testo, Nixon a Roma (1969), in un assurdo teatro che amalgama l'ufficialità politica ed ecclesiastica, il cibo, lo champagne, discorsi ufficiali, la musica moderna e classica, in quel vortice di occhi che incrociano e guardano altri occhi:

[...] Avremo i Corazzieri, un porporato,

le già Eccellenze e i massimi garanti

della Costituzione,

il consommè allo Sherry, il salmone, gli asparagi

da prendere con le molle, il Roederer brut,

i discorsi, gli interpreti, l'orchestra

che suonerà la Rapsodia in blu

e per chiudere Jommelli e Boccherini.

$[\ldots]$

(...)

Può darsi che il banchetto sia differito. Ma

ai toast sorgiamo in piedi coi bicchieri

e ci guardiamo in volto.[...].

(Nixon a Roma, in Montale 1980, p. 676, vv. 9-16 e 23-25). 


\section{IV-La vita con le cose}

L'esempio portato dalla grande poesia di Montale, Senatore a vita della Repubblica italiana nel 1967 e premio Nobel per la letteratura nel 1975, apre l'ingresso, nei testi di poesia degli anni Sessanta e Settanta del Novecento, di cose anche della vita famigliare, che hanno a volte la funzione di creare una tensione tra materialità indifferente e riflessione esistenziale sulla vita quotidiana, sul lavoro domestico ed impiegatizio, sulla libertà dal lavoro e sulla funzione dello stesso lavoro, sul tempo personale, privato e pubblico. Un esempio arriva dalla poesia di Giovanni Giudici (1924-2011), come in questo testo intitolato Le tue ore migliori dalla raccolta La vita in versi (1965), che immette il lettore direttamente a tavola nella vita di famiglia tra le cose e i pensieri di tutti i giorni. Ecco allora tutta una sfilata di oggetti: la tavola apparecchiata, il buon cibo, le stoviglie, i rifiuti raccolti in un piatto, il lavandino, i sughi, fili di pasta, bucce, la tovaglia chiazzata di vino. E su questo sfondo quotidiano di cose, la riflessione del soggetto sul proprio tempo quotidiano (e quello della moglie), sul deterioramento dell'esistenza, che non sembra più appartenergli, poiché al lavoro non si fornisce più solo l'opra (l'opera), ma l'individuo stesso. ${ }^{11}$

II

Dice decoro la tavola apparecchiata,

possiamo avere tutto quel che vogliamo:

all'opulenza mancano forse i fiori.

Il buon cibo conforta dopo l'onesta fatica.

Ma già si ammucchiano stoviglie mentre mangiamo

troppo avidamente, per fare presto.

E ricominci: i necessari rifiuti

in un sol piatto raccogli, riempi

il lavandino ove galleggiano sughi,

affondano fili di pasta, bucce. Adempi

la tua virtù necessaria, riordini

ancora una volta la casa. Io ad altro

lavoro attendo, al mio ufficio, sperando

di fornir l'opra e non me, anzi che giunga la sera,

per godermi la luce residua e, di me

stesso padrone, qualche ora d'avanzo.

Ma non sarà quella la vita vera:

sono queste ore migliori e non ci appartengono.

Eccoci ancora intorno alla mensa serale,

tra le risse dei figli allegramente spietate:

e nuovamente si guasta la linda cucina,

la tovaglia è chiazzata di vino. "Lascia

così - suggerisco - penserai domattina

a tutto. Adesso resta un poco con me».

${ }^{11}$ Si vedano i saggi di Franco Fortini 1965, Giansiro Ferrata 1966 e Fernando Bandini, Introduzione (1975) a Giudici 1991, pp. 453-465. 
(Le tue ore migliori, in Giudici 1991, p. 68, vv. 25-48).

Gli oggetti poveri della quotidianità si ritrovano anche in un grande poeta del secondo Novecento come Giorgio Caproni (1912-1990). Qui si è confrontati con una proposta diversa, che sembra offrire le potenzialità dell'oggetto come coprotagonista dell'esperienza di vita del soggetto. Ad esempio, nella raccolta Il franco cacciatore (1982) il poeta riprende più volte degli oggetti d'uso, il bicchiere e la bottiglia, che diventano fonti di irradiazione di significati ulteriori.

Guardava il bicchiere. Fisso.

Quasi da ridurlo in schegge.

Sapeva che il bicchiere dura

più di chi in mano lo regge?

(All'osteria in Caproni 1989, p. 467).

Ansava sul suo violino.

Stonava. Allegro con moto.

Si può, in un bicchiere vuoto,

bere il ricordo del vino?

(Gelicidio in Caproni 1989, p. 480).

Bicchiere dopo bicchiere.

D'un bel rosso.

Acceso.

In fiamma con la trasparenza

dell'albero.

È solo

(è sera) al tavolo

d'uscio dell'osteria.

Guarda la via andar via

verso il bosco e il buio.

Sa l'ombra.

Ma è in allegria.

Carezza la bottiglia

con mano amorosa.

(Beve vino, o una rosa?)

(Delizia (e saggezza) del bevitore, in Caproni 1989, p. 525).

Il bicchiere, la bottiglia e altri oggetti, la cui funzione è di contenere, potenzialmente, dell'altro, si offrono ad alimentare la percezione di un altrove. Remo Bodei osserva come l'interpretazione della «brocca», di un contenitore atto ad ospitare bevande, assuma in tre pensatori del secolo scorso aspetti estremamente produttivi: «Georg Simmel inaugura nel 1911 una tradizione che si prolungherà, nel 1918, con Ernst Bloch e culminerà, nel 1958, con Martin Heidegger. Pur partendo da differenti premesse e giungendo a differenti conclusioni, tutti e tre considerano dei comuni manufatti, come una coppa, un vaso o una brocca, quali crocevia di relazioni che non riducono la cosa né al suo necessario supporto materiale, né al concetto scheletrico che la definisce» (Bodei 2009, p. 43 e tutto il capitolo In forma di brocca, pp. 42-49). In sostanza, prendendo a prestito e riassumendo Bodei, la «brocca», come qui il bicchiere e la bottiglia nei testi di Caproni sono un crocevia di relazioni, irradiano rapporti che ne moltiplicano la percezione; il bicchiere o la 
bottiglia può essere interpretato come un prolungamento della mano «l'inorganico s'innesta cioè sull'organico come un'altra vita che si somma alla vita animale» (Bodei 2009, p. 43), così come il bicchiere o la bottiglia non avrebbero senso se non rinviassero all' acqua o al vino, e quindi anche ad una sorgente o a un vigneto, alla terra. Inoltre, seguendo l'idea del «pensiero rammemorante», la loro presenza è in relazione ad un tavolo su cui poggiano, al legno, all'albero e al bosco da cui proviene, e all'osteria che li ospita, con i colori del locale e l'atmosfera presente. Secondo Bodei, distinguendo ancora l'oggetto dalla cosa, l'oggetto è privo di quell'aura che caratterizza invece la cosa, di quell'ulteriore rinvio di relazioni e senso: «l'aura è [...] la percezione dell' inafferrabilità e dell' eccedenza di senso della cosa, che dispiega i sui contenuti, erogandoli in misura crescente a chi la considera, ma restando inesauribile nella sua profondità» (Bodei 2009, p. 49).

Nella poesia italiana degli ultimi decenni del Novecento si possono quindi ritrovare cataloghi di oggetti, il soggetto sperso tra gli oggetti, «oggetti orfani», ma anche «soggetti orfani» di oggetti, soggetti posti di fronte ad oggetti quotidiani con i quali viene istaurato un rapporto che li trasforma in cose, secondo il suggestivo studio di Remo Bodei, oppure oggetti che rimangono tali e che interrogano il soggetto. Un altro poeta fondamentale per la poesia italiana del secondo Novecento è il già citato Vittorio Sereni (1913-1983), brillante recensore di Montale e, come Montale, attento lettore della poesia di Guido Gozzano: nel 1936 Sereni si laurea infatti con una tesi in Estetica sulla poetica di Gozzano, che ebbe come relatore il filosofo Antonio Banfi. Nella poesia del primo Sereni ${ }^{12}$ non è difficile ritrovare la presenza di oggetti, di tracce di ascendenza montaliana o comunque oggetti legittimati ad apparire per effetto dei testi de Le occasioni. Infatti, nella prima raccolta di Sereni del 1941, Frontiera, si trova la presenza di una torpediniera (che discende direttamente dalla petroliera della Casa dei doganieri di Montale) e poi di semafori, autocarri e locomotive (Blasucci 2002, p. 65).

Siamo tutti sospesi

a un tacito evento questa sera

entro quel raggio di torpediniera

che ci scruta poi gira se ne va.

(Terrazza in Sereni 1995, p. 32, vv. 6-9).

Qui il traffico oscilla

sospeso alla luce

dei semafori quieti.

(Nebbia in Sereni 1995, p. 18, vv. 1-3).

e tu trasali al rombo

degli autocarri che mordono la montagna

(Soldati a Urbino in Sereni 1995, p. 24, vv. 18-19).

Un fioco tumulto di lontane

locomotive verso la frontiera

(Inverno a Luino in Sereni 1995, p. 31, vv 25-26).

${ }_{12}$ Ci si permette di rinviare a Stefano Sasso 2002 e all'apparato bibliografico di pp. 323-346. 
Nella poesia di Sereni, come anche in quella di Montale, con la modernizzazione della società aumenta la nominazione di oggetti funzionali alla vita quotidiana, ecco allora il telefono, in Comunicazione interrotta (Il telefono / tace da giorni e giorni) (Sereni 1995, p. 104, vv. 1-2) e i televisori, in La poesia è una passione? (e tutta Italia sulle piazze / nei viali e nei bar ferma ai televisori...) (Sereni 1995, p. 153, vv. 11-12).

È però interessante osservare che l'attenzione problematica di Sereni alle cose sembra concentrarsi soprattutto nelle ultime due raccolte, come in questo testo, $I l$ muro da Gli strumenti umani del 1965, dove si ritrova un muro (che separa i morti dai vivi, l'invisibile dal visibile), che pare ricordare la già citata muraglia / che ha in cima cocci aguzzi di bottiglia di Montale.

[...]

Scagliano polvere e fronde scagliano ira

quelli di là dal muro-

e tra essi il più caro. «Papà-faccio per difendermi

puerilmente-papà...».

Non c'è molto da opporgli, il tuffo

di carità il soprassalto in me quando leggo

di fioriture in pieno inverno sulle alture

che lo cerchiano là nel suo gelo al fondo,

se gli porto notizie delle sue cose

se le sento tarlarsi (la duplice

la subdola fedeltà delle cose:

capaci di resistere oltre una vita d'uomo

e poi si sfaldano trasognandoci anni o momenti dopo)

su qualche mensola

in Via Scarlatti 27 a Milano.

(Il muro, in Sereni 1995, p. 179, vv. 14-28).

Oggetti a volte spenti, capaci però di «disegnare» la figura di una persona e rianimarla nel ricordo, nel caso il poeta Umberto Saba, in Saba:

Berretto pipa bastone, gli spenti

oggetti di un ricordo.

Ma io li vidi animati indosso a uno

ramingo in un'Italia di macerie e di polvere.

(Sereni 1995, p. 136, vv. 1-4).

$\mathrm{Ma}$ anche oggetti, cose, presenze che producono smarrimento e inquietudine, come in Le ceneri:

Che spero io più smarrito tra le cose.

Troppe ceneri sparge attorno a sé la noia,

la gioia quando c'è basta a sé sola.

(Sereni 1995, p. 119, vv. 9-11).

Già il titolo della terza raccolta di Sereni, Gli strumenti umani, sembra non solo ricordare gli oggetti concreti che servono al soggetto per vivere, ma materializza gli stessi concetti e pensieri con i quali l'individuo tenta di catturare la realtà e fissarla stabilmente. In Ancora sulla strada di Zenna, le cose si possono «indovinare» 
(la carrucola nel pozzo, la spola della teleferica nei boschi, la lenza buttata a vuoto nei secoli), nel senso che ormai la loro presenza è un fatto acquisito, si sa che appartengono a ciò che non muta, a ciò che si riconosce anche solo per abitudine, a ciò che si ripete sempre identico.

E io potrò per ciò che muta disperarmi

portare attorno il capo bruciante di dolore.

Ma l'opaca trafila delle cose

che là dietro indovino: la carrucola nel pozzo,

la spola della teleferica nei boschi,

i minimi atti, i poveri

strumenti umani avvinti alla catena

della necessità, la lenza

buttata a vuoto nei secoli,

le scarse vite, che all'occhio di chi torna

e trova che nulla nulla è veramente mutato

si ripetono identiche [...].

(Ancora sulla strada di Zenna, in Sereni 1995, p. 113, vv. 12-23).

Nel Sereni della seconda metà del Novecento, l' «assedio delle cose» (come dichiarato da Montale nell'intervista sopra citata) sembra sommarsi alla «ripetizione dell'esistere» (cfr. Mengaldo [1972] 1996, pp. 382-410; Pagnanelli 1980), quindi una specie di doppio assedio: gli oggetti sembrano spesso emergere come marcature interrogative, per ricordare la vita quotidiana, soprattutto lavorativa, che si ripete identica in gesti, azioni, abitudini, e nella ripetizione dell'uguale la vita quotidiana trova la sua lenta estinzione, la sua morte. Gli oggetti si fanno testimonianza del perimetro in cui la vita è vissuta e quotidianamente ripetuta. Per questo gli oggetti spesso non comunicano più nulla o definiscono e ricordano solamente lo spazio e il tempo del soggetto come punto dell'inutile ripetizione del già visto, oggetti che rimangono semanticamente irrelati, pure nominazioni. Esemplarmente si può considerare Posto di lavoro (cfr. anche il commento in Sereni 1993, pp. 108-109), dalla quarta raccolta, Stella variabile del 1981, che si riferisce alla sede lavorativa di Sereni alla casa editrice milanese Mondadori, dove dal 1958 al 1975, sarà direttore editoriale, dopo essere stato per anni dirigente alla Pirelli:

Quei gradini dove fa gomito la scala, tutta

quella gente passata (e ripassata ogni giorno

per lavoro) svoltando dalla scala dalla vita.

Logoro

di quei reiteranti il tappeto in quel punto

a un freddo riflesso di luce. Sia inverno sia estate

e là si fredda

nell'agguato di un pensiero sempre simile a sé

sempre previsto per quel punto

sempre pensato uguale

lo sguardo che là invariabilmente cade

a ogni giorno a ogni ora

di anni di lavoro di anni luce

difreddo-come sempre 
là comincia un autunno.

(Posto di lavoro, in Sereni 1995, p. 192).

Un riflesso (freddo riflesso di luce del tappeto) anche nel senso di specchio, in cui il soggetto vede nell'oggetto se stesso, ossia vede se stesso ripetere quotidianamente le stesse azioni, il ripetersi dell'identico, che lo ha trasformato in una specie di automa, in pura funzione del sistema sociale del lavoro. E nello stesso tempo, in questo prossimo testo, Altro posto di lavoro, le cose dell'esistenza, che fin dal titolo (Altro) si ripetono, sembrano ricordare che la loro vita supera quella del soggetto, impongono la loro durata sull'illusoria supremazia del soggetto, evidenziando la relazione di asincronia esistente tra soggetto e oggetto (come già nel verso: cose / capaci di resistere oltre una vita d'uomo nel testo Il muro, sopra citato).

Non vorrai dirmi che tu

sei tu o che sono io.

Siamo passati come passano gli anni.

Altro di noi non c'è qui che lo specimen

anzi l'imago perpetuantesi

a vuoto-

e acque ci contemplano e vetrate,

ci pensano al futuro: capofitti nel poi,

postille sempre più fioche

multipli vaghi di noi quali saremo stati.

(Altro posto di lavoro, in Sereni 1995, p. 253).

La nuova sede della casa editrice Mondadori, trasferita da via Bianca di Savoia a Milano a Segrate nel 1975, periferia di Milano, realizzata dall'architetto brasiliano Oscar Niemeyer, è un edificio che appare sospeso, in cui dominano l'acqua e le vetrate. Il soggetto, confrontato in un gioco di rispecchiamenti dell'ultra modernità architettonica, sembra catturato da un tempo che lo proietta costantemente in un altrove temporale (capofitti nel poi) e appare moltiplicato e nello stesso tempo ridotto ad esempio da guardare (specimen), a pura immagine (imago) e a piccola annotazione (postille).

Non sorprende allora che la poesia dell'ultimo Sereni arrivi alle soglie della pura nominazione di oggetti (reti, scafo e apparentemente anche Quell'uomo) alla registrazione dell'esistente, che si fissa nelle parole e nient'altro, producendo, come il titolo della poesia, una Fissità.

Da me a quell'ombra in bilico tra fiume e mare

solo una striscia di esistenza

in controluce dalla foce.

Quell'uomo.

Rammenda reti, ritinteggia uno scafo.

Cose che io non so fare. Nominarle appena.

Da me a lui nient'altro: una fissità.

Ogni eccedenza andata altrove. O spenta.

(Fissità, in Sereni 1995, p. 236).

Presenze sempre a fianco o di fronte al soggetto, gli oggetti e le cose sono presenti nella vita di ogni individuo con la loro esistenza variabile. E Sereni ne ricorda anche le potenzialità sorprendenti e inattese, come nell'ultima poesia di Stella variabile dal 
titolo Altro compleanno, nella speranza che un'ardesia propaghi il colore dell'estate (Sereni 1995, p. 266, v. 11). Oppure come quando le persone o gli eventi a noi vicini hanno smesso di «parlare», sono le cose e i ricordi in esse conservati a iniziare a raccontare o a conservare ciò che è stato. È così che tutto questo materiale, le cose, le nature morte, gli oggetti inanimati, esattamente come i morti che hanno smesso di parlare, possono continuare a testimoniare e a raccontare in altri modi, ciò che è stato, ciò che siamo: calce e cenere che possono diventare movimento e luce ${ }^{13}$ Così come nei versi di La spiaggia:

$[\ldots]$

I morti non è quel che di giorno

in giorno va sprecato, ma quelle

toppe di inesistenza, calce o cenere

pronte a farsi movimento e luce.

Non

dubitare,-m'investe della sua forza il mare-

parleranno. ${ }^{14}$

Funding Open Access funding enabled and organized by Projekt DEAL.

Open Access This article is licensed under a Creative Commons Attribution 4.0 International License, which permits use, sharing, adaptation, distribution and reproduction in any medium or format, as long as you give appropriate credit to the original author(s) and the source, provide a link to the Creative Commons licence, and indicate if changes were made. The images or other third party material in this article are included in the article's Creative Commons licence, unless indicated otherwise in a credit line to the material. If material is not included in the article's Creative Commons licence and your intended use is not permitted by statutory regulation or exceeds the permitted use, you will need to obtain permission directly from the copyright holder. To view a copy of this licence, visit http://creativecommons.org/licen ses/by/4.0/.

\section{References}

AA. VV. (2001). Motori diVersi. Intr. di D. Piccini. Milano: Crocetti.

Accademia della Crusca (2017/2018). Manifesti Futuristi (in rete: https://futurismo.accademiadellacrusca .org/index.asp). Accessed 1 Aug 2020.

aut aut (1985). No. 206-207: L'errare dell'identità, marzo-giugno.

Bandini, F. (1975). Introduzione. In G. Giudici (1991), Poesie (1953-1990) (pp. 462-465). Milano: Garzanti.

Blasucci, L. (2002). Gli oggetti di Montale. Bologna: il Mulino.

Bodei, R. (2002). Destini personali. L'età della colonizzazione delle coscienze. Milano: Feltrinelli.

Bodei, R. (2009). La vita delle cose. Roma e Bari: Laterza.

Bodei, R. (2015). Generazioni. Età della vita, età delle cose. Roma e Bari: Laterza.

Bonfiglioli, P. (1958). Pascoli, Gozzano, Montale e la poesia dell'oggetto. Il Verri, 2(4), 34-54.

Caproni, G. (1989). Poesie (1932-1986). Milano: Garzanti.

Carducci, G. (1973). Tutte le poesie. Milano: Bietti.

\footnotetext{
13 Si veda anche, per il significato dei ricordi, Silvia Sereni, Un mondo migliore. Ritratti, Bompiani, Firenze-Milano 2019.

14 La spiaggia, in Sereni 1995, p. 184, vv. 9-14. Si veda Pier Vincenzo Mengaldo 1994, pp. 411-414; Mengaldo 1997 e Mengaldo 1999.
} 
Contini, G. (1974). Una lunga fedeltà. Scritti su Eugenio Montale. Torino: Einaudi.

De Maria, L. (Ed.). (1973). Marinetti e il Futurismo, un'antologia. Milano: Mondadori.

de Rogatis, T. (2011). Le occasioni (1939). Milano: Oscar Mondadori.

Eliot, T. S. (1921). Hamlet and his problems. The sacred wood: essays on poetry and criticism. New York: Knopf.

Ferrata, G. (1966). Poesia e Verbo essere. Giudici, 1991, 457-461.

Fortini, F. (1965). Una nota su Giudici. Giudici, 1991, 453-456.

Giudici, G. (1991). Poesie (1953-1990). Milano: Garzanti.

Gozzano, G. (1977). Poesie. Milano: Rizzoli.

Jacomuzzi, A. (1978). La poesia di Montale Dagli «Ossi» ai «Diari». Torino: Einaudi.

Luperini, R. (1992). Storia di Montale. Roma e Bari: Laterza.

Marinetti, F. T. (1915). Guerra sola igiene del mondo. Milano: Edizioni futuriste di «Poesia».

Mengaldo, P. V. (1989). Quaderno montaliano. Padova: Liviana.

Mengaldo, P. V. (1994). Storia della lingua italiana. Il Novecento. Bologna: il Mulino.

Mengaldo, P. V. (1996). La tradizione del Novecento. Prima serie (1975). Torino: Bollato Boringhieri

Mengaldo, P. V. (2000). La tradizione del Novecento. Quarta serie. Torino: Bollati Boringhieri.

Montale, E. (1976). Sulla poesia. Milano: Mondadori.

Montale, E. (1980). L'opera in versi (Ed., R. Bettarini e G. Contini). Torino: Einaudi.

Montale, E. (1996a). Il secondo mestiere. Arte, musica, società (Ed., G. Zampa). Milano: Mondadori.

Montale. E. (1996b). Il secondo mestiere. Prose 1920-1979 (Ed., G. Zampa). Milano: Mondadori.

Montale, E. (2009). Satura (Ed., R. Castellana). Milano: Oscar Mondadori.

Montale, E. (2010). Diario del '71 e del '72 (Ed., M. Ghezzi). Milano: Oscar Mondadori.

Montale, E. (2011). Le occasioni (Ed., T. de Rogatis). Milano: Oscar Mondadori.

Pagnanelli, R. (1980). La ripetizione dell'esistere. Milano: Scheiwiller.

Pasqualotto, G. (2018). Alfabeto Filosofico. Venezia: Marsilio.

Pedroni, M. (2001). Poesia ciclistica delle origini: Betteloni, Cannizzaro, Gozzano, Pascoli, Stecchetti. Versants. Rivista svizzera delle letterature romanze, 40, 185-205. (in rete: https://www.e-periodica. ch/cntmng?pid=ver-001:2001:40::356). Accessed 1 Aug 2020.

Romolini, M. (2012). Commento a «La bufera e altro» di Montale. Firenze: University Press.

Sasso, S. (2002). Vittorio Sereni tra canzoniere e diario Alla riscoperta di un percorso poetico (19351952). Hamburg: Kovač.

Sasso, S. (2005). Inettitudine e superomismo, ironia e indifferenza ne I colloqui di Guido Gozzano. In H. Krauss, C. Losfeld, K. Van der Meer, \& A. Wortmann (Eds.), Psyche und Epochennorm (pp. 325-340). Heidelberg: Winter.

Savoca, G. (1995). Vocabolario della poesia italiana del Novecento. Le concordanze delle poesie di Govoni, Corazzini, Gozzano, Moretti, Palazzeschi, Sbarbaro, Rebora, Ungaretti, Campana, Cardarelli, Saba, Montale, Pavese, Quasimodo, Pasolini, Turoldo. Bologna: Zanichelli.

Sereni, S. (2019). Un mondo migliore. Ritratti. Milano: Bompiani.

Sereni, V. (1973). Letture preliminari. Padova: Liviana.

Sereni, V. (1993). Poesie. Un'antologia per la scuola (Ed., D. Isella e C. Martignoni). Luino: Nastro e Nastro.

Sereni, V. (1995). Poesie (Ed., D. Isella). Milano: Mondadori.

Sgarbi, V. (2018). Il Novecento. Dal Futurismo al Neorealismo (Vol. I). Milano: Nave di Teseo.

Publisher's Note Springer Nature remains neutral with regard to jurisdictional claims in published maps and institutional affiliations. 